\title{
INTER-RELATIONS AMONG THE FOUR PRINCIPAL TYPES OF ORDER*
}

\author{
BY \\ EDWARD V. HUNTINGTON
}

The four types of order whose inter-relations are considered in this paper may be called, for brevity, (1) serial order; (2) betweenness; (3) cyclic order; and (4) separation.

We first recapitulate the known sets of postulates which define each of these types as an abstract system, and recall the usual geometric interpretation of each type; we then develop the way in which each of these four types may be defined in terms of each of the other three. (For convenience of reference, the numbering of the postulates in earlier publications has been retained.)

1. Serial order. A system $(K, R)$, where $K$ is a class of elements $A, B$, $C, \cdots$, and $R(A B)$, or simply $A B$, is a dyadic relation, is called a "system of serial order" when and only when the following four postulates are satisfied.

In each of these postulates it is understood that distinct letters represent distinct elements of $K$. [The notation " $=0$ " means "is false"; the "horseshoe", $\supset$, means "If ... then"; the "wedge," $v$, means "or" (in the sense of "at least one"); and the "dot," . , means "and." Dots, singly or in groups, serve also as punctuation marks.]
Postulate D. $A A=0$.
Postulate I. $A B \vee B A$.
(“Irreflexiveness.")
Postulate II. $A B \cdot B A:=0$.
("Connexity.")
("Asymmetry.")
Postulate IV. $A B . \supset . X B \vee A X$. ("Inclusiveness.")

From properties II and IV, the following property is deducible as a theorem:

Postulate III. $A B \cdot B C$. . . $A C$. ("Transitivity for distinct elements.") $A C$.)

(Proof. By IV, $A B$. د. $C B \vee A C$. But $C B$ conflicts with $B C$, by II. Hence

Also, IV is a consequence of III and I.

(Proof. By I, $X B \vee B X$. But if $B X$, then by III, $A B . B X$. ᄀ . $A X$. Hence $X B \vee A X$.)

Hence the following alternative sets of postulates are equivalent, and

* Presented to the Society, April 20, 1935; received by the editors September 27, 1934. 
either of them may be taken as a set of independent postulates for serial order*:
(1) D, I, II, III;
(2) D, I, II, IV.

Geometrically speaking, this "abstract" system $(K, R)$ of serial order may be represented by a "concrete" system in which $K$ is the class of points on a directed straight line (that is, a straight line having a definite "sense" indicated by an arrow), and $R(A B)$ means "the point $A$ precedes the point $B$ " when the line is traversed in the direction of the arrow. Or briefly: serial order is the order of points on a directed straight line.

2. Betweenness. A system $(K, R)$, where $K$ is a class of elements $A, B$, $C, \cdots$, and $R(A B C)$, or simply $A B C$, is a triadic relation, is called a system of "betweenness" when and only when the following five postulates are satisfied. (In each of these postulates, beyond $\mathrm{D}$, it is understood that distinct letters represent distinct elements of $K$.)

Postulate D. If $A B C$ is true, then $A, B, C$ are distinct.

Postulate B. $B A C \vee C A B \vee A B C \vee C B A \vee A C B \vee B C A$.

Postulate A. $A B C . \supset . C B A$.

Postulate C. $A B C \cdot A C B:=0$.

Postulate 9. $A B C . \supset . A B X \vee X B C$.

From these five properties, the following eight properties are deducible as theorems:

Postulate 1. $X A B \cdot A B Y . \supset . X A Y$.

Postulate 2. $X A B . A Y B . \supset . X A Y$.

Postulate 3. $X A B \cdot A Y B . \supset . X Y B$.

Postulate 4. $A X B . A Y B . \supset . A X Y \vee A Y X$.

Postulate 5. $A X B \cdot A Y B$. ว. $(A X Y \vee Y X B)$. ( $A Y X \vee X Y B)$.

Postulate 6. $X A B . Y A B . \supset . X Y B \vee Y X B$.

Postulate 7. $X A B . Y A B . \supset . X Y A \vee Y X A$.

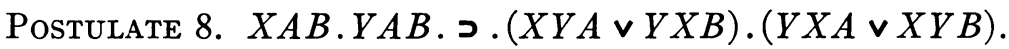

* Sets of postulates for serial order date back at least as far as the early work of G. Peano and B. Russell. [Partial references will be found in E. V. Huntington's $A$ complete sel of postulates for the theory of absolute continuous magnitude, these Transactions, vol. 3 (1902), pp. 264-279; or, Complete existential theory of the postulates for serial order, Bulletin of the American Mathematical Society, vol. 23 (1917), pp. 276-280; or The Continuum and Other Types of Serial Order, second edition, Harvard University Press, 1917.] In Set 1, if Postulate III (the law of transitivity for distinct elements) is replaced by a law of transitivity for all elements, then Postulate II becomes redundant, being a consequence of this extended law of transitivity and Postulate D. In the present paper, Postulate IV is introduced for the sake of its analogy with Postulates 9 and 10, below. 
The following twelve sets of postulates are equivalent, and any one of them may be taken as a set of independent postulates for betweenness*:

(1) A, B, C, D, 1, 2.

(5) A, B, C, D, 1, 8.

(9) A, B, C, D, 3, 4, 6 .

(2) A, B, C, D, 1, 5 .

(6) A, B, C, D, 2, 4 .

(10) A, B, C, D, 3, 4, 7 .

(3) A, B, C, D, 1, 6 .

(7) A, B, C, D, 2, 5 .

(11) A, B, C, D, 3, 4, 8 .

(4) A, B, C, D, 1, 7 .

(8) A, B, C, D, 3, 5 .

(12) A, B, C, D, 9.

The most familiar concrete example of this abstract system of betweenness is the system $(K, R)$ in which $K$ is the class of points on andirected straight line, and $R(A B C)$ means " $B$ is between $A$ and $C$ " in the geometric sense. (This is the concrete example from which the abstract system takes its name.) In brief, "betweenness" is the order of points on an undirected straight line.

3. Cyclic order. A system $(K, S)$, where $K$ is a class of elements $A, B$, $C, \cdots$, and $S(A B C)$, or simply $A B C$, is a triadic relation, is called a system of "cyclic order" when and only when the following five postulates are satisfied. (In each of these postulates, beyond D, it is understood that distinct letters represent distinct elements of $K$.)

Postulate D. If $A B C$ is true, then $A, B, C$ are distinct.

Postulate $\mathrm{B}^{\prime}$. The system contains at least one true triad, say $X Y Z$.

Postulate E. $A B C . \supset . B C A$.

Postulate C. $A B C . A C B:=0$.

Postulate 9. $A B C . \supset . A B X \vee X B C$.

From these five properties, the following three properties are deducible as theorems:

Postulate B. $A B C \vee B C A \vee C A B \vee C B A \vee B A C \vee A C B$.

Postulate 2. $X A B . A Y B$. ว. $X A Y$.

Postulate 3. $X A B . A Y B . \supset . X Y B$.

The following four sets of postulates are equivalent, and any one of them

* Sets 1-11 were given by E. V. Huntington and J. R. Kline, Sets of independent postulates for betweenness, these Transactions, vol. 18 (1917), pp. 301-325. Set 12 was given by E. V. Huntington, $A$ new set of postulates for betweenness with proof of complete independence, ibid., vol. 26 (1924), pp. 257-282. (This latter paper includes a discussion of certain peculiarities of Postulates 5 and 8 , and an analysis of the significance of E. H. Moore's concept of complete independence.) W. E. Van de Walle, On the complete independence of the postulates for betweenness, ibid., vol. 26 (1924), pp. 249-256, shows that each of the Sets $1-10$ is completely independent, and that Set 11 is not. 
may be taken as a set of independent postulates for cyclic order:*
(1) B, C, D, E, 2.
(3) B, C, D, E, 9.
(2) B, C, D, E, 3 .
(4) $\mathrm{B}^{\prime}, \mathrm{C}, \mathrm{D}, \mathrm{E}, 9$.

Cyclic order is represented geometrically by a class $K$ of points on a directed closed line, with $S(A B C)$ meaning "the arc running from $A$ through $B$ to $C$, in the direction of the arrow, is less than one complete circuit." In brief, cyclic order is the order of points on a directed closed line.

When necessary to distinguish between the two triadic relations, $R(A B C)$ for betweenness, and $S(A B C)$ for cyclic order, the prefixes $R$ and $S$ will be retained.

4. Separation. A system $(K, R)$, where $K$ is a class of elements $A, B$, $C, \cdots$, and $R(A B C D)$, or simply $A B C D$, is a tetradic relation, is called a system of "separation of pairs," or simply a system of "separation," when and only when the following six postulates are satisfied. (In each of these postulates, beyond $\mathrm{D}$, it is understood that distinct letters represent distinct elements of $K$.)

Postulate D. If $A B C D$ is true, then $A, B, C, D$ are distinct elements of $K$.

Postulate $\mathrm{F}^{\prime}$. The system contains at least one true tetrad, say $X Y Z W$.

Postulate G. $A B C D . \supset . B C D A$.

Postulate H. $A B C D \cdot A B D C:=0$.

Postulate $\mathrm{R}^{\prime}$. At least one true tetrad is reversible; that is, if there is any true tetrad, then there is at least one true tetrad $A B C D$ such that $D C B A$ is also true.

Postulate 10. $A B C D . \supset . A X C D \vee A B C X$.

* See E. V. Huntington, Sets of completely independent postulates for cyclic order, Proceedings of the National Academy of Sciences [Washington], vol. 10 (1924), pp. 74-78. Another definition of cyclic order (in terms of a tetradic relation) is referred to in the next footnote.

Set (4) is new, and requires a proof of $\mathrm{B}$ from $\mathrm{B}^{\prime}, \mathrm{E}, \mathrm{C}, 9$, which proceeds as follows:

By $\mathrm{B}^{\prime}$, there is one true triad, say $U V W$. By $9, U V W . \supset . A V W \vee U V A$.

If $U V A$, then by $\mathrm{E}, V A U$, whence by $9, V A U$. つ.WAUVVAW, whence by $\mathrm{E}, U W A \vee A W V$. Therefore $A V W \vee U W A \vee A W V$.

But if $U W A$, then by $9, U W A . \supset . V W A \vee U W V$, where $U W V$ conflicts with $U V W$ by $\mathrm{C}$, and $V W A . \supset . A V W$ by E. Therefore $A V W \vee A W V$, whence by $\mathrm{E}, V W A \vee W V A$. We have thus proved (a) $U V W . \supset . V W A \vee W V A$,

where $U V W$ represents any true triad.

Case 1. If $V W A$, then by (a), $V W A . \supset . W A B \vee A W B$, whence by $\mathrm{E}, W A B \vee W B A$. But if $W A B$, then by (a), $W A B$. ᄀ. $A B C \vee B A C$, and if $W B A$, then by (a), $W B A . \supset . B A C \vee A B C$.

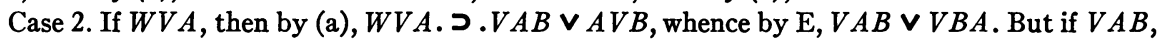
then by (a), $V A B . \supset . A B C \vee B A C$, and if $V B A$, then by (a), $V B A . \supset . B A C \vee A B C$.

Hence in any case, $A B C \vee B A C$. Hence by E, $A B C . B C A . C A B: \vee: B A C . A C B . C B A$, from which $\mathbf{B}$ follows at once. 
From these six properties the following eleven properties are deducible as theorems:

Postulate F. If $A, B, C, D$ are distinct elements of $K$, then at least one of the twenty-four tetrads $A B C D, A B D C, \cdots, D C B A$ is true.

Postulate R. Every true tetrad is reversible; that is, $A B C D .>$. $D C B A$.

Postulate 11. $A B X C . A B C Y$. ว . $A B X Y$.

Postulate 12. $A B X C . A B C Y$. ᄀ. $B X C Y$.

Postulate 13. $A B X C . A B C Y$. ว . $A X C Y$.

Postulate 14. $A B C X . A B C Y$. ว . $A B X Y \vee A B Y X$.

Postulate 15. $A B C X . A B C Y$. ᄀ. $A C X Y \vee A C Y X$.

Postulate 16. $A B C X . A B C Y$. ว. $B C X Y \vee B C Y X$.

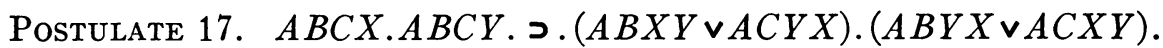

Postulate 18. $A B C X . A B C Y$. ว. $(A B X Y \vee B C Y X)$. $(A B Y X \vee B C X Y)$.

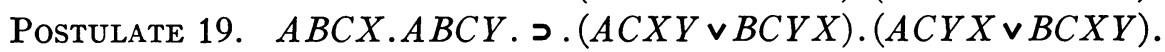

The following ten sets of postulates are equivalent, and any one of these may be taken as a set of independent postulates for separation*:

(1) D, F, G, H, R, 10 .

(6) D, F, G, H, R, 11, 16.

(2) D, F, G, H, R, 12 .

(7) D, F, G, H, R, 11, 17.

(3) D, F, G, H, R, 13.

(8) D, F, G, H, R, 11, 18.

(4) D, F, G, H, R, 11, 14.

(9) D, F, G, H, R, 11, 19.

(5) D, F, G, H, R, 11, 15.

(10) $\mathrm{D}, \mathrm{F}^{\prime}, \mathrm{G}, \mathrm{H}, \mathrm{R}^{\prime}, 10$.

A geometrical example of a system of "separation" is the system $(K, R)$ in which $K$ is a class of points on an undirected closed line, and $R(A B C D)$ means "the pair of points $A, C$ is separated by the pair $B, D$." In brief, "separation" treats of the order of points on an undirected closed line.

In the language of modern geometry, "separation" is the theory of order on the "projective line" (the so-called "straight line" of projective geometry). It may be suggested, in passing, that the concept of the "fourth harmonic point" determined by three given points on such a projective line is a concept which it would be interesting to define by the postulational method.

* See E. V. Huntington and K. E. Rosinger, Postulates for separation of point-pairs (reversible order on a closed line), Proceedings of the American Academy of Arts and Sciences [Boston], vol. 67 (1932), pp. 61-145. On p. 70 of this paper the following corollary is established: In every system which satisfies Postulates D, F', G, H, 10, we have either (R) Every true tetrad is reversible; or else (S) Every true tetrad is non-reversible; and on p. 63 it is noted that if we introduce

Postulate $S^{\prime}$. At least one true tetrad is non-reversible,

then Postulates D, F', G, H, 10, $\mathrm{S}^{\prime}$ will define the theory of non-reversible order on a closed line (just as Postulates D, $\mathrm{F}^{\prime}, \mathrm{G}, \mathrm{H}, 10, \mathrm{R}^{\prime}$ define the theory of reversible order on a closed line). This theory of non-reversible order on a closed line is essentially the same as the theory of cyclic order, expressed in terms of a tetradic instead of a triadic relation. 
The inter-relations among these four types of order may be classified under four headings.

$$
\$ \$ 1.1-1.5
$$

Under the first heading, we show that each of the other three types may be defined directly in terms of serial order; and also that separation may be defined directly in terms of each of the other three types. The details of the proofs require nothing more than a checking up of all the possible cases for each of the postulates involved, and will be left to the reader.

1.1. Betweenness defined in terms of serial order. In a given system of serial order, three elements $A, B, C$ will stand in the "betweenness" relation $A B C$ when $A B$ and $B C$ are true, and also when $C B$ and $B A$ are true, but not otherwise. That is, in the system of serial order we may define the relation of betweenness as follows:

$$
A B C::=:: A B \cdot B C: \vee: C B \cdot B A \text {. }
$$

The triadic relation thus defined is readily shown to satisfy all the postulates A, B, C, D, 9 for betweenness.

1.2. Cyclic order defined in terms of serial order. Similarly, in a given system of serial order the relation of cyclic order may be defined as follows:

$$
A B C::=:: A B . B C: \vee: B C . C A: \vee: C A . A B \text {. }
$$

The triadic relation thus defined satisfies all the postulates B, C, D, E, 9 for cyclic order.

1.3. Separation defined in terms of serial order. Again, in a given system of serial order, four elements $A, B, C, D$ will stand in the "separation" relation, $A B C D$, under conditions expressed by the following definition:

$$
\begin{aligned}
& A B C D::=:: A B \cdot B C . C D: \vee: B C . C D \cdot D A: \vee: C D . D A . A B: \vee \\
& : D A \cdot A B \cdot B C: \vee: D C \cdot C B \cdot B A: \vee: A D \cdot D C . C B: \vee \\
& : B A . A D . D C: \vee: C B . B A . A D \text {. }
\end{aligned}
$$

The tetradic relation thus defined satisfies all the postulates $D, F^{\prime}, G, H, R^{\prime}$, 10 for separation.

1.4. Separation defined in terms of betweenness. Suppose now we have a given system of betweenness. The relation of separation may be defined in this system as follows:

$$
A B C D::=:: A B C . B C D: \vee: B C D . C D A: \vee: C D A . D A B: \vee: D A B . A B C \text {. }
$$

1.5. Separation defined in terms of cyclic order. Again, if we have a given system of cyclic order, the relation of separation may be defined in that system as follows:

$$
A B C D::=:: A B C . C D A, \vee . A D C . C B A \text {. }
$$


$\S \S 2.1-2.3$

Under the second heading we consider definitions which are not absolute, but involve a reference to an arbitrarily selected element of the given system, say $Z$.

2.1. Serial order defined in terms of cyclic order, with respect to $Z$. In a given system of cyclic order, if we exclude any arbitrarily chosen element $Z$, the remaining elements may be arranged in serial order (with respect to $Z$ ) by the following definition:

$$
A B:=: Z A B \text {. }
$$

The element $Z$ itself may then be brought into the series, if desired, by defining $A Z$ as true and $Z A$ as false.

2.2. Betweenness, $R(A B C)$, defined in terms of cyclic order, with respect to $Z$. In a given system of cyclic order, if we exclude any arbitrarily chosen element $Z$, we may define the betweenness relation $R(A B C)$ among the remaining elements as follows:

$$
R(A B C)::=:: Z A B . B C Z: \vee: Z C B . B A Z \text {. }
$$

The element $Z$ itself may then be brought into the betweenness system, if desired, by defining $R(A B Z)$ and $R(Z B C)$ as true and $R(A Z C)$ as false.

2.3. Betweenness defined in terms of separation, with respect to $Z$. Suppose now the given system is a system of separation. Then if we exclude an arbitrary element $Z$, we may define the betweenness relation among the remaining elements as follows:

$$
A B C:=: Z A B C \text {. }
$$

The element $Z$ itself may then be brought into the betweenness system, if desired, by defining $A B Z$ and $Z B C$ as true and $A Z C$ as false.

$$
\S \S 3.1-3.2
$$

Under the third heading, the definitions are also not absolute, but involve a reference to an arbitrarily chosen pair of elements, say $U$ and $V$, in the given system.

3.1. Serial order defined in terms of betweenness, with respect to $U, V$. In a given betweenness system, let $U, V$ be any two selected elements. Then all the elements may be arranged in serial order (with respect to $U, V$ ) by the following definitions (where, as usual, distinct letters denote distinct elements):

$U V$ true; and $V U$ false.

$A U:=: A U V . \quad A V:=: A U V \vee U A V . \quad U A:=: U A V \vee U V A$.

$V A:=: U V A$.

$A B::=:: A U V . A B V: \vee: A U V . A V B: \vee: U A V . U A B: \vee: U V A . U A B$. 
3.2. Cyclic order, $S(A B C)$, defined in terms of betweenness, with respect to $U, V$. In a given betweenness system, let $U, V$ again be any two arbitrarily chosen elements. Then we may define the relation of cyclic order among the elements of this system (with respect to $U, V$ ) as follows*:

$$
\begin{aligned}
& S(A U V) . S(U V A) . S(V A U)::=:: A U V \vee U V A \text {. } \\
& S(A V U) . S(V U A) . S(U A V)::=:: U A V \text {. } \\
& S(A B U) . S(B U A) . S(U A B):: \\
& =:: A B U . B U V: \vee: B U V . B U A: \vee: U A V . U A B: \vee: U V A . U A B \text {. } \\
& S(A U B) . S(U B A) . S(B A U):: \\
& =:: A U V . A U B: \vee: U B V . U B A: \vee: U V B . U B A: \vee: U A B . A U V . \\
& S(A B V) . S(B V A) . S(V A B): \text { : } \\
& =:: A U V . A B V: \vee: U A B . A B V: \vee: A V B . A V U: \vee: U V A . U A B \text {. } \\
& S(A V B) . S(V B A) . S(B A V): \text { : } \\
& =:: A V B . U V B: \vee: U V B . U B A: \vee: B U V . B A V: \vee: U B A . B A V \text {. } \\
& S(A B C) . S(B C A) . S(C A B): \text { : } \\
& =:: B C A . B C U . B U V: \vee: B C A . B U C . B U V: \vee: U C A . U B C . U B V: \\
& \mathrm{v}: U C A . U A B . U C V: \mathrm{v}: U A B . U B C . U A V: \vee: A U B . A B C . A U V: \\
& \vee: A B U . A U V . A B C: \vee: C U A . C A B . C U V: \vee: C A U . C U V . C A B: \\
& \vee: U V B . V B C . B C A: \vee: U V A . V A B . A B C: \vee: U V C . V C A . C A B \text {. } \\
& \$ \$ 4.1-4.2
\end{aligned}
$$

Under the fourth heading, the definitions involve reference to three arbitrary elements of the given system. In $\$ 4.1$, one of the three reference elements, say $Z$, is distinguished from the other two, say $U, V$. In $\$ 4.2$, the three reference elements, say $U, V, W$, are coordinate.

4.1. Serial order defined in terms of separation, with respect to $Z$ and $U, V$. Given a separation system, in which two elements $U$ and $V$ are arbitrarily selected as reference elements. Then if a third arbitrary element $Z$ is excluded from the system, all the remaining elements may be arranged in serial order (with respect to $U, V$ ) by the following definitions:

$U V$ true; and $V U$ false.

$$
\begin{aligned}
A U:= & : Z A U V . U A:=: Z U A V \vee Z U V A . \\
A V:= & : Z A U V \vee Z U A V . \quad V A:=: Z U V A . \\
A B::= & : Z Z A U V . Z A B U: \vee: Z A U V . Z A U B: \vee: Z U A V . Z U A B: \\
& \vee: Z U V A . Z U A B .
\end{aligned}
$$

The element $Z$ itself may then be brought into the series, if desired, by defining $U Z, V Z, A Z$ as true and $Z U, Z V, Z A$ as false.

* For assistance in formulating and verifying the definitions under $\$ \$ 3.2$ and $4.2, \mathrm{I}$ am indebted to Mr. B. Notcutt. 
4.2. Cyclic order defined in terms of separation, with respect to $U, V, W$. Given a separation system, in which three elements, $U, V, W$, are arbitrarily selected as reference elements ("anchorage points"). The relation of cyclic order (with respect to $U, V, W$ ) may be thus defined in this system:

$U V W . V W U . W U V$ true; and $W V U . U W V . V U W$ false.

$A U V . U V A . V A U:=: A U V W \vee A W U V$.

$A V W . V W A . W A V:=: A V W U \vee A U V W$.

$A W U . W U A . U A W:=: A W U V \vee A V W U$.

$A V U . V U A . U A V:=: A V W U$.

$A W V . W V A . V A W:=: A W U V$.

$A U W . U W A . W A U:=: A U V W$.

$U A B . A B U . B U A:=: A V W U . A B V U: \vee: A V W U . A V B U:$

$\vee: A W U V . A B U V: \vee: A U V W . A B U V$.

$V A B \cdot A B V . B V A:=: A W U V . A B W V: \vee: A W U V \cdot A W B V:$

$\vee: A U V W . A B V W: \vee: A V W U . A B V W$.

$W A B . A B W . B W A:=: A U V W . A B U W: \vee: A U V W . A U B W:$

$$
\vee: A V W U . A B W U: \vee: A W U V . A B W U \text {. }
$$

$A B C::=:: A U V W . A B V W . A B C W: \vee: B U V W . B C V W . B C A W:$

$\vee: C U V W . C A V W . C A B W: \vee: A U V W . A B V W . A B W C:$

$\vee: B U V W . B C V W . B C W A: \vee: C U V W . C A V W . C A W B$.

Summary. These results may be summarized in the following table, in which $Z, U, V, W$ denote arbitrarily selected elements of the given system.

\begin{tabular}{l|l|l}
\hline \hline & The relation of & may be defined within a given system of \\
\cline { 2 - 3 } 1.1 & Betweenness & serial order. \\
1.2 & Cyclic order & serial order. \\
1.3 & Separation & betweenness. \\
1.4 & Separation & cyclic order. \\
\hline 1.5 & Separation & cyclic order, with respect to $Z$. \\
\hline 2.1 & Serial order & cyclic order, with respect to $Z$. \\
2.2 & Betweenness & separation, with respect to $Z$. \\
\hline 3.3 & Betweenness & betweenness, with respect to $U, V$. \\
\hline 3.2 & Serial order & betweenness, with respect to $U, V$. \\
\hline 4.1 & Cyclic order & separation, with respect to $Z$ and $U, V$. \\
4.2 & Serial order & separation, with respect to $U, V, W$. \\
\hline
\end{tabular}

HARVARD UNIVERSITY, Cambridge, Mass. 